Stevanato, F.; Nielsen, A. W.; Sumer, B. Mutlu; Fredsoe, J.

Flow Velocities and Bed Shear Stresses in a Stone Cover under an Oscillatory Flow

Verfügbar unter / Available at:

https://hdl.handle.net/20.500.11970/100286

Vorgeschlagene Zitierweise / Suggested citation:

Stevanato, F.; Nielsen, A. W.; Sumer, B. Mutlu; Fredsoe, J. (2010): Flow Velocities and Bed Shear Stresses in a Stone Cover under an Oscillatory Flow. In: Burns, Susan E.; Bhatia, Shobha K.; Avila, Catherine M. C.; Hunt, Beatrice E. (Hg.): Proceedings 5th International Conference on Scour and Erosion (ICSE-5), November 7-10, 2010, San Francisco, USA. Reston, Va.: American Society of Civil Engineers. S. 609-618. 


\title{
Flow Velocities and Bed Shear Stresses in a Stone Cover under an Oscillatory Flow
}

\author{
F. Stevanato ${ }^{1}$, A. W. Nielsen ${ }^{2}$, B. M. Sumer ${ }^{3}$ and J. Fredsøe ${ }^{4}$
}

${ }^{1}$ Technical University of Denmark, DTU Mekanik, Section of Coastal, Maritime and Structural Engineering, Building 403, 2800 Kgs. Lyngby, Denmark; e-mail: Francesco.stevanato09@imperial.ac.uk. Present address: Imperial College London, Department of Civil and Environmental Engineering, London SW7 2AZ, UK

2 Technical University of Denmark, DTU Mekanik, Section of Coastal, Maritime and Structural Engineering, Building 403, 2800 Kgs. Lyngby, Denmark; e-mail: awni@mek.dtu.dk

${ }^{3}$ Technical University of Denmark, DTU Mekanik, Section of Coastal, Maritime and Structural Engineering, Building 403, 2800 Kgs. Lyngby, Denmark; e-mail: bms@mek.dtu.dk

${ }^{4}$ Technical University of Denmark, DTU Mekanik, Section of Coastal, Maritime and Structural Engineering, Building 403, 2800 Kgs. Lyngby, Denmark; e-mail: jf@mek.dtu.dk

\begin{abstract}
In order to get a better understanding of the interaction between the waveinduced, near-bed oscillatory flow, the stone cover and the sea bed, physical model tests were carried out. The tests were conducted in an oscillating water tunnel. The bottom of the tunnel was covered by one, two and three layers of stones. The flow velocities in the pores of the stones were measured using LDA (Laser Doppler Anemometer). In addition to the velocity measurements, the bed shear stresses were also measured using a hotfilm (Constant Temperature Anemometry).

It is found that the boundary layer of the outer flow penetrates quite a substantial amount of thickness into the stone cover, depending on the flow conditions above. Below this level the velocity remains constant. The level of turbulence in between the stones is found to be very high: 3 to 4 times higher than turbulence level over a ripple covered bed in steady current boundary layer without any externally generated turbulence. The bed shear stress is found to be very low, more than ten times smaller than in the case of a smooth base bottom without stone cover.
\end{abstract}

\section{INTRODUCTION}

Stone covers have been used for scour protection of offshore structures for decades. This was also the case for many of the offshore wind farms erected during 
the last decade. These wind farms are located in shallow waters and some of them are exposed to high waves and strong currents. Under these extreme conditions the scour protection of the wind turbines suffered unacceptable damages. One of the reasons for these damages to the scour protection could be the heavy wave action.

The purpose of the present study is to gain a better understanding of the interaction between the wave-induced near-bed flow and a stone cover, with the focus on the flow through the pores of the stone cover layer. A significant amount of flow and turbulence was measured for all three cases, namely one, two, and three layers of stones, covered in the tests.

\section{SETUP}

\section{General description}

The experiments were conducted in an oscillatory water tunnel with a rectangular working section of $29 \mathrm{~cm}$ height and $39 \mathrm{~cm}$ width. The horizontal working section of the oscillator is $10 \mathrm{~m}$. Both ends of the horizontal part of the oscillator are connected to a vertical riser. One of the risers is open, while the other is closed and connected to a pneumatic system. During the experiments the bottom of the working section was covered with crushed stones. One, two and three layers of stones were tested. A sketch of the setup is shown in Figure 1.

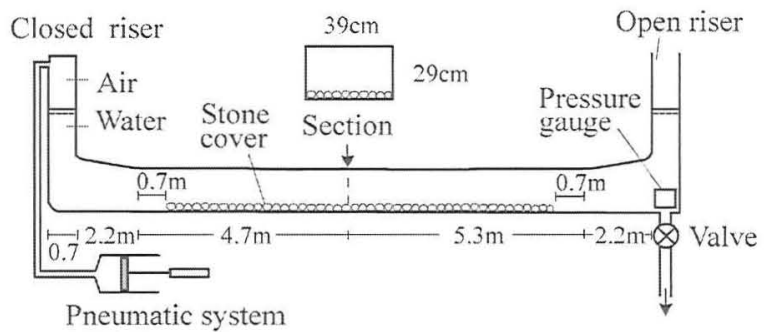

Figure 1 Sketch of the oscillating water tunnel.

The velocities were measured using a two component Laser Doppler Anemomenter (LDA). The LDA system consisted of a $300 \mathrm{~mW}$ argon-ion laser exciter, Dantec Dynamics LDA-04 System, Dantec Dynamics 55H21 Frequency tracker, Dantec Dynamics 55N11 Frequency shifter and a laser probe with focal length of $310 \mathrm{~mm}$ (in air). To record the direction of the flow a pressure cell was placed at the bottom of the open riser.

The velocity measurements were made through the transparent side wall of the tunnel. The velocities above the stone layer were measured at the center line of the tunnel to reduce the effects of secondary flows due to the rectangular cross 
section of the tunnel. It was not possible to measure at the center line in the pores of the stone layer as the stones between the LDA probe and the pores at the center line would block the laser beams. For this reason the velocities in the pores between the stones were measured adjacent to the side wall. The sample frequency for the velocities and pressure measurements was $70 \mathrm{~Hz}$, and 40 waves were recorded at each measuring point.

Three experiments have been conducted measuring the bed shear stress under three layers of stones. A one-component Dantec hot-film probe was used to measure the bed shear stress. It was mounted flush with the base bottom, and located $15.4 \mathrm{~cm}$ from the side wall, at the test section, and calibrated using a calibration channel. A detailed description of the measurement technique is given in Sumer et al. (1993).

The stones had a mean height of $k=36 \mathrm{~mm}$ with a standard deviation of $9 \mathrm{~mm}$. The height was measured by taking a random sample of 50 stones placed on the bottom of the tunnel, then the stone height was measured as the vertical distance from the bottom of the tunnel to the top of the stones.

\section{Horizontal Position of the Velocity Measurements}

The velocity measurements in the pores between the stones are affected by the horizontal position of the measurements. Nevertheless, measurements at the vertical line through the center of the pore would be a sensible option. However, due to the irregularities of the stones, the center of the pore is very hard to define. For this reason the "centroid" of the pore is defined, as in the following (Figure 2):

1. The points of contact $\mathrm{A}$ and $\mathrm{B}$ between the horizontal projection of two adjacent stones and the side wall of the tunnel are identified.

2. The location of point $C$ : The middle point between points $A$ and $B$, is found.

3. The horizontal line through point $\mathrm{C}$, perpendicular to the line between $\mathrm{A}$ and $\mathrm{B}$, intersects the horizontal projection of the two stones at point $\mathrm{D}$.

4. The centroid $\mathrm{P}$ is defined as the middle point between points $\mathrm{C}$ and $\mathrm{D}$.

The velocity was measured across the vertical line passing through point $\mathrm{P}$. Twenty different pores were measured.

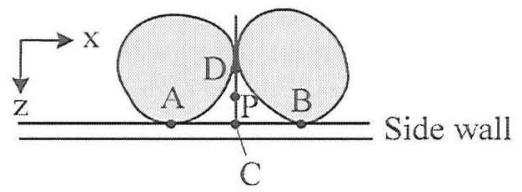

Figure 2 Definition of the centroid, plan-view. 


\section{TEST CONDITIONS}

The maximum free stream velocity in the experiment was limited by the stability of the stones. The stones were stable for velocities smaller than approximately $1.1 \mathrm{~m} / \mathrm{s}$; free stream velocities larger than this would cause movements of the stones which were unacceptable for the experiment. To ensure stability for the stones a free stream velocity of approximately $1.0 \mathrm{~m} / \mathrm{s}$ was used for the experiments $(1.0 \mathrm{~m} / \mathrm{s}$ for one layer of stones, $0.95 \mathrm{~m} / \mathrm{s}$ for two layers of stones and $0.96 \mathrm{~m} / \mathrm{s}$ for three layers of stones).

The wave period was governed by the natural frequency of the oscillation in the tunnel, as a wave period very different from the natural period of the tunnel would "contaminate" the velocity signal. The natural period of the flume was measured to be between $9.73 \mathrm{~s}$ and $10.02 \mathrm{~s}$ for no stones and 3 layers of stones, respectively. As the difference between the natural periods is small, the wave period was kept constant at $T=9.73 \mathrm{~s}$ for all the experiments.

\section{RESULTS}

The velocities are phase and "pore" averaged; first the velocities are averaged over $N_{w}=40$ wave periods:

$$
\widetilde{u}(y, \omega t)=\frac{1}{N_{w}} \sum_{n=1}^{N_{w}} u(y, \omega(t+(n-1) T))
$$

where $u$ is the measured horizontal, streamwise velocity in position $\mathrm{P}$ (see Figure 2) and elevation $y$ (fixed), $\omega$ is the angular frequency of the oscillatory flow and $t$ is the time. Then they are averaged over the $N_{p}=20$ measured pores:

$$
U(y, \omega t)=\frac{1}{N_{p}} \sum_{m=1}^{N_{p}} \tilde{u}(m, y, \omega t)
$$

$\omega t=0^{\circ}$ is defined as the zero-up-crossing in the streamwise velocity in the field above the stones.

The phase- and pore-averaged horizontal velocities for three layers of stones are shown in Figure 3. The velocities are almost constant, for the particular phases, up to 6 to $7 \mathrm{~cm}$ above the base bottom then they increase towards the top of the stone cover. The region between $y=6-7 \mathrm{~cm}$ and the top of the stones is influenced by the outer flow. The constant velocity layer is independent of the boundary, regardless of the number of stone layers. There will of course be a thin boundary layer at the base bottom.

There is a slight difference in the magnitude of the positive and the negative horizontal velocities. This is caused by the steady streaming induced by the convergent/divergent flow at the inlets from the risers, see Sumer et al. (1993a). This 
is also influencing the vertical velocities.

Due to the rectangular shape of the cross section of the tunnel, a secondary flow will appear in the cross section causing an upward-directed flow adjacent to the side wall. These secondary flows are directly linked to the streamwise flow; the larger the streamwise flow, the larger the secondary flows. As seen in Figure 4 there is a general upward directed flow in the pores. However, this is larger for the phases from $\omega t=0^{\circ}$ to $\omega t=90^{\circ}$ than for the phases of $\omega t=180^{\circ}$ to $\omega t=250^{\circ}$. The reason is that the streamwise velocity is larger for the phases from $\omega t=0^{\circ}$ to $\omega t=90^{\circ}$ than from $\omega t=180^{\circ}$ to $\omega t=250^{\circ}$, as seen in Figure 3 .

The phase- and pore-averaged horizontal velocities for two layers of stones are shown in Figure 5. As in the case of three layers of stones, the boundary layer flow over the stone cover in the outer flow penetrates into the stone cover over a depth of $4 \mathrm{~cm}$ below which the velocity remains constant, the constant velocity layer.

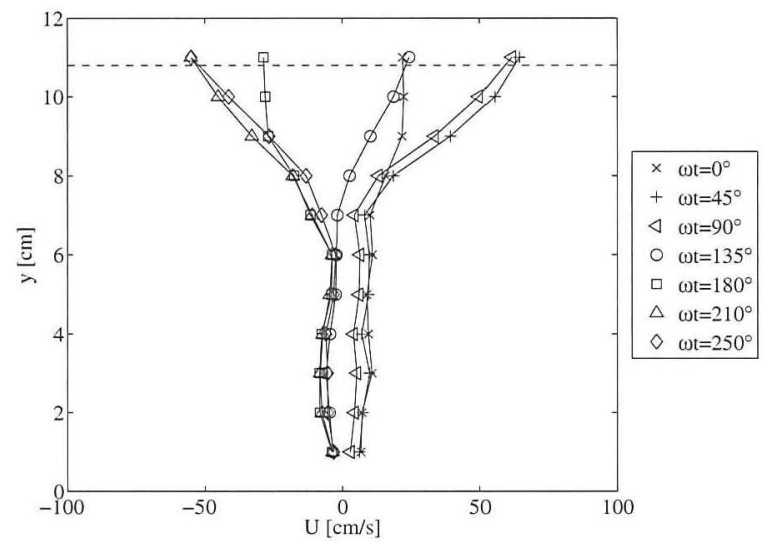

Figure 3 The velocity profiles of horizontal phase- and pore-averaged velocities.

Three layers of stones. Dashed line: The average height of the stones.

The phase- and pore-averaged horizontal velocities for one layer of stones are shown in Figure 6. As in the cases of three and two layers of stones the outer boundary layer penetrates into the stone layer. In this case down to $1 \mathrm{~cm}$ above the base bottom. This shows that the constant velocity layer has become rather thin and it is expected, in the analogy with the two and three layer cases. 


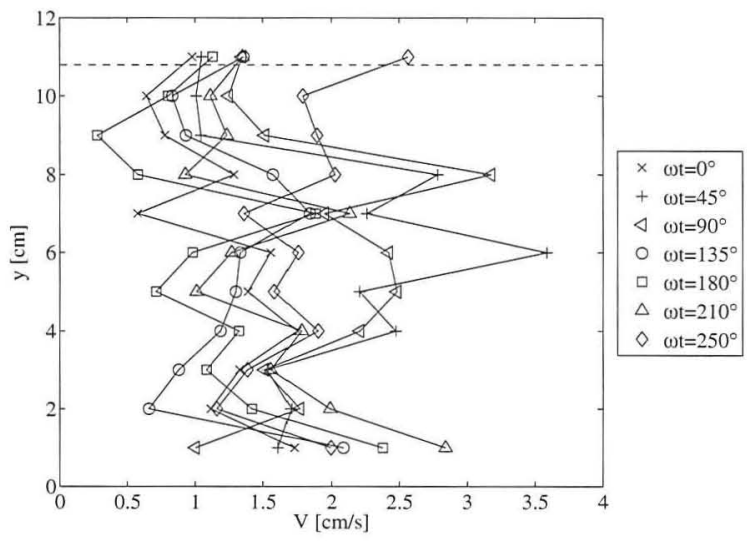

Figure 4 The velocity profiles of vertical phase- pore-averaged mean velocities.

Three layers of stones. Dashed line: The average height of the stones.

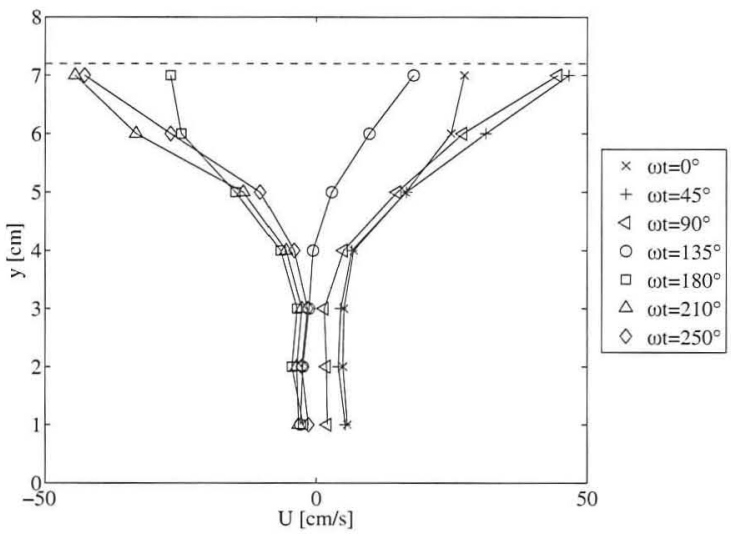

Figure 5 The velocity profiles of horizontal phase- and pore-averaged mean velocities. Two layers of stones. Dashed line: The average height of the stones.

As seen from Figure 3, Figure 5 and Figure 6, the horizontal velocities are significantly larger in the case of three layers of stones than in the case of one and two layers of stones. The level of turbulence is not influenced by this effect. 


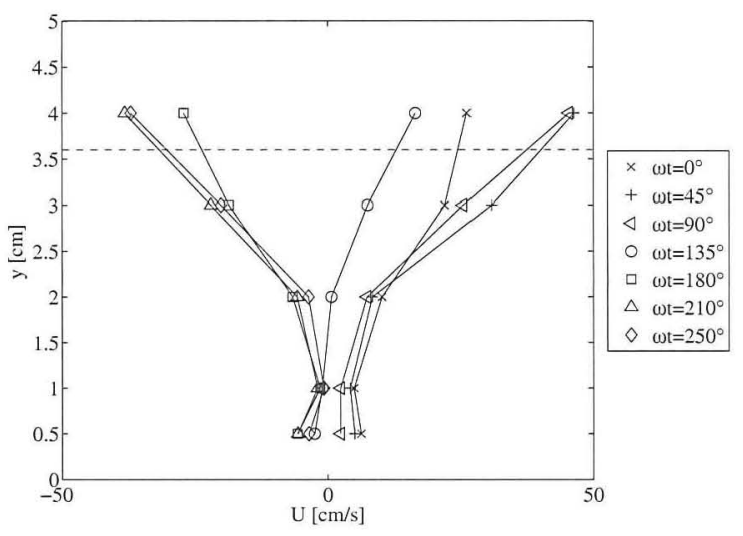

Figure 6 The velocity profiles of horizontal phase- and pore-averaged mean velocities. One layer of stones. Dashed line: The average height of the stones.

The phase- and pore-averaged horizontal turbulent velocities for three layers of stones are shown in Figure 7. The turbulent velocity is defined as:

$$
U_{s}(y, \omega t)=\frac{\sum_{i=1}^{N_{p}} \sqrt{\left[u^{\prime}(i, y, \omega t)\right]^{2}}}{N_{P}}
$$

where $N_{P}$ is the number of pores, $u^{\prime}$ is the measured oscillatory component of the horizontal velocity and $y$ is the vertical position. The turbulent velocities are very large over the entire stone cover. At the top of the stone cover they are between 7 and $12 \mathrm{~cm} / \mathrm{s}$ decreasing to 2.5 to $4 \mathrm{~cm} / \mathrm{s} 1 \mathrm{~cm}$ above the base bottom. The turbulent velocities are almost constant in the constant velocity layer.

The phase-average of the bed shear stresses under three layers of stones are shown in Figure 8. The bed shear stresses were measured in three different layouts of the stones. The bed shear stresses measured in pore 1 and 2 were measured in the middle of the horizontal projection of a pore. In the case of pore 3 there was an offset in the streamwise direction. As seen in the figure the bed shear stress in pore 3 has a peak between $250^{\circ}$ and $310^{\circ}$ there is around 4 to 5 times higher than the bed shear stress measured in the two other pores. The reason for this can be the offset of the measuring position or the actual layout of the stones around the measuring point.

The bed shear stress is varying between 0.06 and $0.97 \mathrm{~cm}^{2} / \mathrm{s}^{2}$. This is a very low bed shear stress. For example Jensen et al. (1989) reported bed shear stresses up to around $10 \mathrm{~cm}^{2} / \mathrm{s}^{2}$ for an undisturbed oscillatory flow over a smooth bed with $U_{m 0}=0.45 \mathrm{~m} / \mathrm{s}$. However, if the critical Shields number is reached for sediment under 
the stone protection a high rate of bed load is expected, due to the high level of turbulence. Sands with grain size of 0.05 to $0.1 \mathrm{~mm}$ will be unstable under the measured bed shear stress, assuming a critical Shields parameter of 0.03 and $s=2.65$. Fig. 14 in Sumer et al. (2003) gives the dimensionless bed load discharge as function of the Shields parameter for different levels of turbulence near the bottom. The dimensionless bed load discharge in Sumer et al. (2003) is defined as:

$$
\Phi_{b}=\frac{q}{\sqrt{g(s-1) d_{50}^{3}}}
$$

where $q$ is the discharge, $g$ is the acceleration due to gravity, $s$ is the specific gravity of the sediment and $d_{50}$ is the mean size of the sediment. The dimensionless level of turbulence near the bottom is in the same publication defined as:

$$
\left(\frac{\sqrt{\overline{u^{\prime 2}}}}{U_{f b}}\right)_{0}
$$

where $U_{f b}$ is the friction velocity at the bottom, $U_{f b}=\sqrt{\tau_{0} / \rho}$.

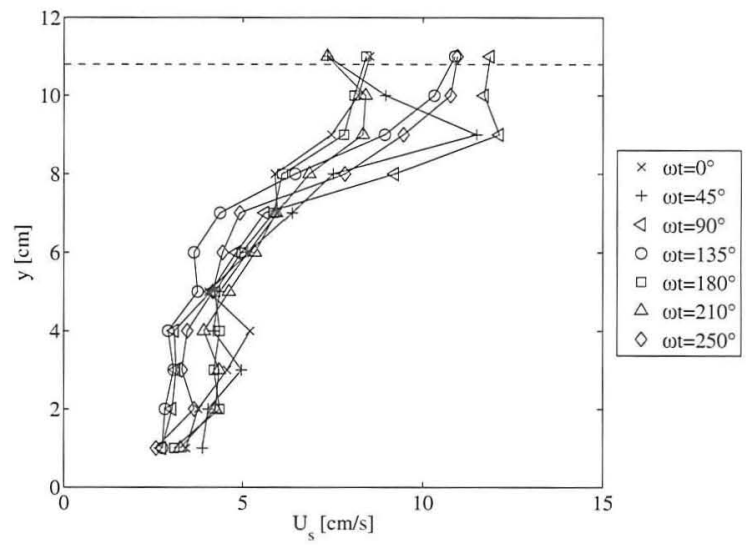

Figure 7 The horizontal phase- and pore-averaged turbulent velocities for different phase values. Three layers of stones.

A representative dimensionless peak level of turbulence will then be around $6\left(\omega t=45^{\circ}, U_{s}=4 \mathrm{~cm} / \mathrm{s}\right.$ and $\left.\tau / \rho=0.5 \mathrm{~cm}^{2} / \mathrm{s}^{2}\right)$, see Figure 7 and Figure 8 . The highest values of the dimensionless turbulence level given in Sumer et al. (2003) are 2.5 to 2.75. Using this and assuming that the Shields parameter reach 0.06 will give a dimensionless bed load of around 0.03 . This is a really high value for so small Shields numbers. A typical dimensionless turbulence level over a ripple covered bed 
in current without any externally generated turbulence will be 1.7 and that will give a dimensionless bed load discharge around ten times smaller.
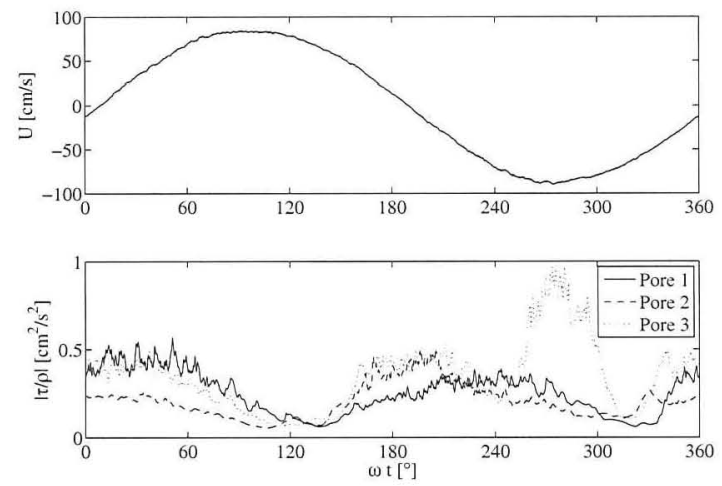

Figure 8 Phase-average of the horizontal free stream velocity and the bed shear stress measured under three layers of stones. Bed shear stresses in three different pores are presented.

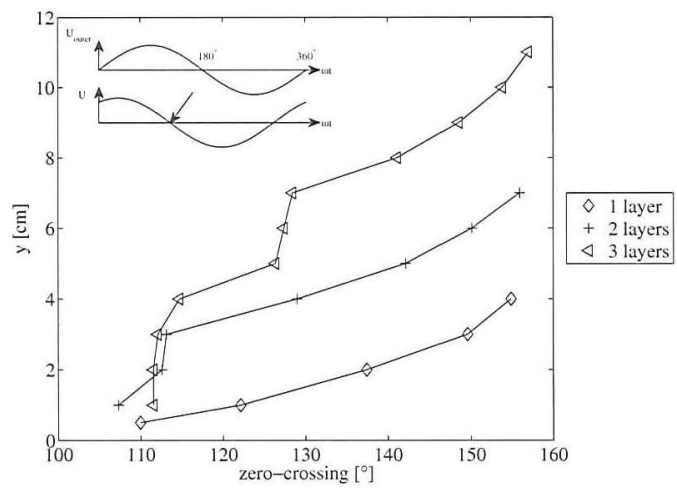

Figure 9 Profiles for zero-down-crossing phase for one, two and three layers of stones.

The zero-down-crossing phase for the three setups is shown in Figure 9. There is a large change in the zero-crossing phase from the top of the stones to the base bottom of around $45^{\circ}$. This is expected as the flow velocities in the stone layer is relatively small compared to the free flow velocity and the flow resistance in the 
stones is large. This means that the momentum of the flow in the stone layer will be "dissipated" faster than the momentum in the free flow when the pressure gradient reverses.

\section{CONCLUSION}

The velocities and turbulence have been measured in the pores of a stone cover under an oscillatory flow. Cases with one, two and three layers of $4 \mathrm{~cm}$ large stones have been tested. In the case of three layers of stones the bed shear stress at the base bottom was also measured. It is found that the boundary layer of the outer flow penetrates into the stone cover over a depth of $4 \mathrm{~cm}$. Below this level the velocity remains constant, the constant velocity layer. The level of turbulence in between the stones is found to be very high: 3 to 4 times higher than turbulence level over a ripple covered bed in steady current boundary layer without any externally generated turbulence. The bed shear stress is found to be very low, more than ten times smaller than in the case of a smooth base bottom without stone cover.

\section{ACKNOWLEDGEMENT}

This research was carried out as part of the Statkraft Ocean Energy Research Program, sponsored by Statkraft (www.statkraft.no). The study was partially supported by the Danish Council for Strategic Research (DSF)/Energy and Environment under the program Seabed Wind Farm Interaction (http:/sbwi.dhigroup.com, sagsnr. 2104-07-0010) and DHI (www.dhigroup.com). During his two years Master course, F. Stevanato was sponsored by "Universita' degli Studi di Padova" and the TIME network of Engineering Schools.

\section{REFERENCES}

Jensen B.L., Sumer B.M. and Fredsøe, J. (1989). "Turbulent oscillatory boundary layers in high Reynolds numbers". J. Fluid Mech., vol. 206, 265-297

Sumer B.M., Chua, L.H.C., Cheng N.-S. and Fredsøe, J. (2003). "Influence of turbulence on bed load sediment transport". J. Hydraulic Eng., Vol. 129, No. 8, 585-596.

Sumer B.M., Arnskov M.M., Christiansen, N. and Jørgensen, F.E. (1993). "Twocomponent hot-film probe for measurements of wall shear stress". Exp. Fluids, vol. 15, 380-384.

Sumer B.M., Laursen, T.S. and Fredsøe, J. (1993a). "Wave boundary layers in a convergent tunnel". Coastal Engineering, vol. 20, 317-342. 